\title{
The Influence of Health Literacy and Depression on Diabetes Self-Management: A Cross-Sectional Study
}

\author{
D. Maneze,${ }^{1,2,3,4}$ B. Everett, ${ }^{1,2,3,4}$ C. Astorga, ${ }^{1}$ D. Yogendran, ${ }^{1}$ and Y. Salamonson ${ }^{2,3,4}$ \\ ${ }^{1}$ South Western Sydney Local Health District, 59a Cumberland Road, Ingleburn, NSW 2565, Australia \\ ${ }^{2}$ School of Nursing and Midwifery, Western Sydney University, Locked Bag 1797, Penrith, NSW 2751, Australia \\ ${ }^{3}$ Centre for Applied Nursing Research, Locked Bag 7103, Liverpool, NSW 1871, Australia \\ ${ }^{4}$ Ingham Institute for Applied Medical Research, P.O. Box 3151, Liverpool, NSW 2170, Australia
}

Correspondence should be addressed to D. Maneze; della.maneze@sswahs.nsw.gov.au

Received 6 May 2016; Revised 15 July 2016; Accepted 19 July 2016

Academic Editor: Bernadette Bartlam

Copyright (C) 2016 D. Maneze et al. This is an open access article distributed under the Creative Commons Attribution License, which permits unrestricted use, distribution, and reproduction in any medium, provided the original work is properly cited.

\begin{abstract}
Despite an increasing focus on health literacy in the clinical setting and in the literature, there is still ongoing debate about its influence on diabetes self-management. The aim of the study was to examine the relationships of sociodemographic, clinical, and psychological factors on health literacy and diabetes self-management. A cross-sectional survey was undertaken on 224 patients with type 2 diabetes at two diabetes centres in Sydney, Australia. Findings showed that people with low health literacy were more likely to (a) have lower educational attainment; (b) be migrants; and (c) have depressed mood. Unexpectedly, those who met $\mathrm{HbA}_{1 \mathrm{c}}$ threshold of good glucose control were more likely to have low health literacy. Predictors of low diabetes self-management included (a) younger age group (AOR: 2.58, 95\% CI: 1.24-4.64); (b) having postsecondary education (AOR: 2.30, 95\% CI: 1.05-5.01); (c) low knowledge of diabetes management (AOR: 2.29, 95\% CI: 1.25-4.20); and (d) having depressed mood (AOR: 2.30, 95\% CI: 1.30-4.06). The finding that depressed mood predicted both low health literacy and low diabetes self-management stresses the importance of screening for depression. Increasing people's understanding of diabetes self-management and supporting those with depression are crucial to enhance participation in diabetes self-management.
\end{abstract}

\section{Introduction}

The rapid rise in the global prevalence of diabetes lends urgency to the need for investigations beyond the walls of traditional factors such as poor nutrition, obesity, and sedentary behaviour. In 2013, diabetes was reported in 382 million people worldwide, a figure projected to increase by $55 \%$ to 592 million in 2035 [1]. Type 2 diabetes mellitus (T2DM) is the most prevalent form affecting $90 \%$ of adults with diabetes [2] and is increasingly being diagnosed in younger age groups [3]. While biochemical and clinical research is important, grassroots level sociocultural research is needed to understand the underlying sociodemographic and cultural environment which influences the self-efficacy of patients to perform the daily tasks of self-managing their chronic condition. For example, among migrants to many developed countries like Australia, acculturation to host culture, language and cultural barriers, and socioeconomic factors contribute to an increased incidence of lifestyle diseases, approximating that of the receiving country [4]. Migrants encounter many personal and systemic barriers in managing chronic conditions like diabetes $[5,6]$, which adds to the complexity of implementing self-management interventions in this population. Understanding how these factors interrelate and influence self-management is important to provide person-centred strategies to enhance the health of people living with diabetes.

Diabetes self-management (DSM) is considered an essential cornerstone of good diabetes control [7]. It is reported to reduce the level of glycated haemoglobin level $\left(\mathrm{HbA}_{1 \mathrm{c}}\right)$, a clinical measure of adequate control, by as much as $37 \%$ [8]. Having a lower $\mathrm{HbA}_{1 \mathrm{c}}$ value $(\leq 7 \%$ or $\leq 53 \mathrm{mmol} / \mathrm{mol}$ ) reduces the likelihood of developing micro- and macrovascular complications over time [9]. Despite the increasing evidence that supports the benefits of DSM, uptake remains low, especially in culturally diverse populations $[10,11]$. Among people with T2DM, knowledge deficit and understanding about diabetes 
and its complications have been found to be low in those with low health literacy [11], posing a barrier to DSM [12]. Given this association, improving health literacy, defined as "the capacity to look for, process and understand health information to make informed decisions" [13] seems an important priority to empower patients to self-manage their diabetes [14]. Paasche-Orlow and Wolf [15] postulated that the mechanisms contributing to poorer outcomes among those with low health literacy include low self-efficacy, lack of access to and utilisation of resources and services, and language and cultural issues in clinical encounters. It is important, however, to acknowledge that socioeconomic and demographic factors such as age, educational level, ethnocultural background, and having conditions that require complex care are underscoring limited health literacy $[6,10,16]$. Low levels of health literacy have been found to be common among patients who are from lower socioeconomic backgrounds and among migrants with limited English language proficiency, the elderly, and those with chronic diseases [17]. While some studies have found that low health literacy is associated with poor diabetes self-management, poor control, and more complications [12], the evidence regarding this association is inconsistent [18]. This could be due to other psychosocial and demographic factors that may affect health literacy and/or differences in measuring this construct [19].

Adding to the complications of suboptimal selfmanagement is reduced psychological well-being [20]. This is a vicious cycle that may further disempower patients. For example, psychological comorbidity, like depression, contributes to lower self-care [21], which in turn leads to poorer health status leading to more depression and comorbidities which further reduce DSM [22].

The aim of the study was to examine the relationships between sociodemographic, clinical, and psychological factors and health literacy and its relationship with DSM within a culturally diverse urban population with T2DM. Specifically, we sought to investigate the relationship between health literacy and other factors influencing DSM. The hypotheses in this study were as follows:

(1) Self-management in patients with T2DM is associated with sociodemographic factors (age, gender, educational level, marital status, and country of birth), clinical factors (self-rated general health, $\mathrm{HbA}_{1 \mathrm{c}}$ ), and psychological factors (depression, confidence, knowledge, and health literacy).

(2) Health literacy in patients with T2DM is associated with sociodemographic factors (age, gender, educational level, marital status, and country of birth), clinical factors (self-rated general health, $\mathrm{HbA}_{1 \mathrm{c}}$ ), and psychological factors (depression, confidence, and knowledge).

\section{Methods}

2.1. Study Design and Setting. We used a cross-sectional design, patients with T2DM attending the diabetes outpatient clinics at two centres in South Western Sydney Australia. The study setting is a culturally diverse region with $52 \%$ of its population born overseas. Of these, 59\% speak a language other than English at home and 13\% are new arrivals, settling in Australia within the last five years [23]. Southwest Sydney is also one of the largest and most rapidly growing districts within the Sydney metropolitan area [24] with approximately $21 \%$ of the population in the low socioeconomic stratum [25] and only about $30 \%$ of its population completing secondary school. Unemployment rates are high, with a mean rate of $8 \%$ (range 5\%-31\%), ranking some of these suburbs (10 out of 38 ) among the most disadvantaged areas in Australia [25].

2.2. Participants and Recruitment. Using convenience sampling, participants were recruited between May and December 2015 from the outpatient diabetes clinics of two large centres in the South Western Sydney Local Health District (SWSLHD). Eligibility criteria included (1) age of 18 years and above; (2) being diagnosed with T2DM; (3) having $\mathrm{HbA}_{1 \mathrm{c}}$ test in the last two years recorded in their clinical file. Patients attending the outpatient clinics for their regular appointment with the diabetes educator, specialists, or dietician were identified and referred to the research team by the clinicians. One of the researchers then explained the purpose of study and sought consent from potential participants. Those who consented to participate in the study were asked to complete a questionnaire. Consent included access to participants' hospital records to retrieve their latest recorded clinical data including $\mathrm{HbA}_{1 \mathrm{c}}$, height, and weight. Researchers measured height and weight of participants to compute for their body mass index (BMI) after they have completed the questionnaire if this was not available in their clinical records.

2.3. Instruments. A pilot study was initially undertaken with 20 participants. The initial questionnaire consisted of 63 questions including items from five validated instruments: the English language acculturation scale [26], PHQ-2 depression scale [27], diabetes knowledge [28], diabetes self-efficacy [29], and diabetes self-management [30]. Results of the pilot testing indicated that participants found the questionnaire to be too complex and lengthy, including those whose first language was English. Following discussion with the research team, a consensus was reached to simplify the questionnaire and reduce the survey to only include 34 items. These were items related to demographic and clinical characteristics, three brief validated measures, namely, the (a) 3-item Health Literacy Scale [31]; (b) 2-item PHQ-2 to assess depressed mood and anhedonia [27]; and (c) 16-item Diabetes SelfManagement Scale [30]. As single item questions have been found to be as valid and reliable as multiple-item scales, particularly when constructs that are being measured are fairly homogenous $[32,33]$ the two standardised scales that measured diabetes self-efficacy and diabetes knowledge were replaced with two single items; namely, "In a scale of 1 to 10 (1 being not confident to 10 being very confident), how confident are you that you will be able to manage your diabetes?" and "In a scale of 1 to 10 (1 being very poor to 10 being excellent), how do you rate your knowledge about diabetes?" Subjective assessment of perceived overall health was likewise assessed with a single question: "In general, how would you describe 
your general health?" with a five-point Likert scale response, excellent, very good, good, fair, and poor.

Cronbach's alpha was calculated for each validated instrument used. This is a measure of the extent to which the items in the questionnaire consistently assess the same idea or concept [34]. The internal consistency is expressed as a numerical value between 0 and 1 with scores between 0.70 and 0.90 indicating good correlation among items in the questionnaire [35].

2.3.1. Brief Health Literacy Scale. Health literacy was evaluated using the 3-item Health Literacy Scale [31] and included the following: (1) How often do you have problems learning about your medical condition because of difficulty understanding written information? and (2) How confident are you filling out forms by yourself? and (3) How often do you have someone help you read hospital materials? Each item was rated with a 5-point Likert scale with lower scores indicating lower health literacy.

2.3.2. Depression Scale. The 2-item Patient Health Questionnaire (PHQ-2) [27] was used to assess anhedonia and depressed mood over a 2-week period. This 4-point Likert scale has been used extensively to determine the presence of depression, with higher scores indicating the presence of depression [36]. A cut-off aggregate score of 2 has been found to have high sensitivity in detecting major depressive disorder (92.7\%) and any depressive disorder (80.4\%), with specificity of $73.7 \%$ and $80.4 \%$, respectively [27].

2.3.3. Diabetes Self-Management Scale. The 16-item Diabetes Self-Management Questionnaire (DSMQ-16) [30] was used in this study because of its brevity relative to other related scales. More importantly it had significantly stronger correlation with $\mathrm{HbA}_{1 \mathrm{c}}$ which is an important measure of diabetes control.

2.3.4. Glycated Haemoglobin $\left(H b A_{1 c}\right)$. The glycated haemoglobin level or $\mathrm{HbA}_{1 \mathrm{c}}$ is recommended in the monitoring of glucose control as it reflects the average blood glucose level over three months and has a good correlation with diabetes complications [37]. A cut-off value of $7 \%$ has been recommended to indicate good control $[37,38]$.

2.4. Analysis. Sample size calculation for the outcome variable was based on low DSM rate of $50 \%$. Taking into account the 11 sociodemographic, clinical, knowledge, and psychological predictor variables as listed in the hypothesis and using the sample size calculation based on Peduzzi et al. [39] of $N=10 \mathrm{k} / \mathrm{p}$ (where $N$ is the minimum number of cases needed, $k$ is the number of predictor variables, and $p$ is the proportion of low DSM rate), the minimum sample size required was 220 .

We used SPSS version 23 software [40] for all data analysis. Frequencies and percentages were computed for categorical variables, and mean, median, and standard deviation and interquartile range were computed for continuous variables. As none of the continuous variables were normally distributed, age, duration of diabetes diagnosis, BMI, medical comorbidities, confidence, knowledge, health literacy, and DSMQ-16 scores were dichotomised at the median. However, the PHQ-2 score was dichotomised at 2 to represent "not depressed" (0-1) and "depressed" (2-6) to be consistent with the high sensitivity of this cut-off shown in previous studies [36]. While dichotomisation of variables may have the disadvantage of loss of analytical power and some important information, it has the benefits of reducing the variability in a skewed data and consequently the random error, making the results more accurate [41]. In addition, it simplifies the results and thus presents findings that are easily understandable to a wide range of audience [42]. Data in this study was skewed with a high variability in the responses. Furthermore, corrective logarithmic transformation calculations performed did not produce findings dissimilar to the dichotomised results obtained.

The Chi-square test was used to assess relationships between two categorical variables, and logistic regression analysis (forward conditional method, with listwise deletion of cases with missing data [43]) was used to identify predictors of depression and predictors of DSM. The variables included in these regression analyses were demographic, clinical, and psychological characteristics of participants as previously described in the hypotheses.

\section{Results}

In total, 275 patients who met the inclusion criteria were approached. Of these 11 refused to participate and 40 were excluded from the final analysis as they were not able to complete the questionnaire and/or they did not have a recorded $\mathrm{HbA}_{1 c}$ in the last two years. Cronbach's alpha for the following instruments used in the study showed good item correlation and internal consistency: Brief Health Literacy Scale $(\alpha=0.83)$; Depression Scale (PHQ-2) $(\alpha=0.88)$; and the Diabetes Self-Management Scale (DSM-16) $(\alpha=0.79)$.

The demographic profile of our sample approximated the statistical profile of the study setting. Of the 224 participants included in the final analysis, 56\% were born overseas and $7 \%$ were newly arrived migrants (less than 5 -year duration of stay in Australia) with $40 \%$ speaking a language other than English at home. Nineteen percent (19\%) of the sample had postsecondary schooling. Table 1 shows the clinical, knowledge, and psychological characteristics of participants. Although the overall health literacy score was high (median: 10; range: $0-12$ ), the overall diabetes knowledge score was lower (median: 7, range: 0 to 10 ). While the overall DSM-16 score was high (median: 35, range: 7 to 47 ), $61 \%$ of the sample were obese (BMI: $\geq 30 \mathrm{kgm}^{2}$ ), and $81 \%$ had an $\mathrm{HbA}_{1 \mathrm{c}}$ over $7 \%$ with $30 \%$ having more than two comorbidities. Fortyseven percent $(47 \%)$ of the participants rated their general health as fair to poor. Fifty percent of the participants had a score of 2 or more in the PHQ-2 suggesting the presence of depressed mood or anhedonia [27, 36]. Those who had PHQ2 score more than 2 were also found to have longer duration of diabetes diagnosis (more than 10 years), more comorbidities (more than 2), lower confidence, and less DSM behaviours. 
TABLE 1: Characteristics of T2DM participants $(n=224)$.

Characteristics

Sociodemographic

Age, median (IQR) years (range: 22-90)

$60(17)$

Sex: male, $n(\%)$

119 (53)

Marital status: with partner, $n(\%)$

147 (66)

Highest educational attainment*

(i) Up to primary schooling, $n$ (\%)

$33(15)$

(ii) Secondary schooling (years 7 to 12 , TAFE, trade), $n$ (\%)

(iii) More than secondary schooling (postgraduate), $n$ (\%)

Clinical characteristics

Self-rated health: fair or poor, $n$ (\%)

Duration of diabetes diagnosis: median (IQR) years (range: 0-46)

Body mass index (BMI): median (IQR) (range: 19.0-64.5)

$32.4(11)$

$\mathrm{HbA}_{1 \mathrm{c}}$ : median (IQR) (range: 4.8-14.0)

Knowledge and psychological factors

Depression: PHQ-2 score: median (IQR) (range: 0-6)

Knowledge about diabetes: median (IQR) (range: 0-10)

${ }^{*}$ Missing data.

TABLE 2: Group comparison of high and low health literacy levels by participant characteristics.

\begin{tabular}{|c|c|c|c|c|}
\hline Characteristics & Low health literacy $(\leq 10)$ & High health literacy $(>10)$ & Unadjusted odds ratio & $p$ \\
\hline \multicolumn{5}{|l|}{ Sociodemographic } \\
\hline Age: $<60$ years, $n(\%)$ & $56(44)$ & $57(58)$ & $0.58(0.34-0.98)$ & 0.042 \\
\hline Sex: male, $n(\%)$ & $65(52)$ & $54(55)$ & $1.15(0.68-1.96)$ & 0.601 \\
\hline Marital status: with partner, $n(\%)$ & $86(68)$ & $61(62)$ & $0.77(0.44-1.34)$ & 0.348 \\
\hline \multicolumn{5}{|l|}{ Highest educational attainment } \\
\hline (i) Up to primary schooling & $27(22)$ & $6(6)$ & $0.24(0.09-0.61)$ & $<0.001$ \\
\hline (ii) Secondary schooling & $81(66)$ & $65(66)$ & $1.09(0.63-1.91)$ & \\
\hline (iii) More than secondary schooling & $15(12)$ & $27(28)$ & $2.81(1.40-5.66)$ & \\
\hline Country of birth: overseas-born, $n(\%)$ & $80(64)$ & $45(46)$ & $0.49(0.29-0.84)$ & 0.009 \\
\hline \multicolumn{5}{|l|}{ Clinical characteristics } \\
\hline Self-rated health: fair or poor, $n(\%)$ & $111(90)$ & $88(90)$ & $0.97(0.41-2.32)$ & 0.946 \\
\hline $\mathrm{HbA}_{1 \mathrm{c}}:$ high $(>7 \%), n(\%)$ & $96(76)$ & $85(87)$ & $2.04(1.00-4.17)$ & 0.047 \\
\hline \multicolumn{5}{|l|}{ Knowledge and psychological factors } \\
\hline Confidence diabetes management: low (up to 8 ), $n(\%)$ & $94(75)$ & $60(61)$ & $1.86(1.05-3.29)$ & 0.032 \\
\hline Knowledge about diabetes: low (up to 7), $n(\%)$ & $83(66)$ & $53(54)$ & $1.64(0.95-2.82)$ & 0.073 \\
\hline Psychological status, depressed (PHQ-2: $\geq 2$ ) & $71(56)$ & $41(42)$ & $0.56(0.33-0.95)$ & 0.031 \\
\hline DSMQ-16 score: low (up to 35$), n(\%)$ & $64(51)$ & $57(58)$ & $0.74(0.44-1.26)$ & 0.283 \\
\hline
\end{tabular}

3.1. Group Comparisons of Low and High Health Literacy. Using the median score of 10 as the cut-off for the Brief Health Literacy scale, group comparisons of sociodemographic, clinical, and knowledge and psychological factors were computed using the Chi-square test. As shown in Table 2, those who were older, had up to primary schooling, were overseas-born, were less confident about diabetes management, and had
PHQ-2 score $\geq 2$, had low health literacy. Surprisingly, those with $\mathrm{HbA}_{1 \mathrm{c}}>7 \%$, indicating poor control, were more likely to have high health literacy $(p=0.047)$.

3.2. Predictors of Low Health Literacy. Using forward stepwise logistic regression analysis, four variables emerged as independent and significant predictors of low health literacy: 
TABLE 3: Predictors of low health literacy in T2DM patients $(n=224)$.

\begin{tabular}{|c|c|c|c|}
\hline Variables & Adjusted odds ratio (95\% CI) & Std error (SE) & $p$ \\
\hline \multicolumn{4}{|c|}{ (i) Highest educational attainment (reference: secondary schooling) } \\
\hline (a) Up to primary schooling & $3.12(1.17$ to 8.30$)$ & 0.50 & 0.023 \\
\hline (b) More than secondary schooling & $0.35(0.16$ to 0.74$)$ & 0.39 & 0.006 \\
\hline (ii) Country of birth: overseas-born & 2.17 (1.21 to 3.91$)$ & 0.30 & 0.010 \\
\hline (iii) Poor glucose control $\left(\mathrm{HbA}_{1 \mathrm{c}}:>7 \%\right)$ & $0.41(0.19$ to 0.90$)$ & 0.40 & 0.026 \\
\hline (iv) Psychological status, depressed (PHQ-2: $\geq 2$ ) & 2.01 (1.12 to 3.59$)$ & 0.30 & 0.019 \\
\hline
\end{tabular}

CI denotes confidence interval.

Nagelkerke $R^{2}=0.191$.

Hosmer-Lemeshow goodness-of-fit for the model: Chi-square $=2.937, \mathrm{df}=7$, and $p=0.891$.

TABLE 4: Predictors of low diabetes self-management in T2DM patients $(n=224)$.

\begin{tabular}{|c|c|c|c|}
\hline Variables & Adjusted odds ratio $(95 \% \mathrm{CI})$ & Std error (SE) & $p$ \\
\hline (i) Age: $\leq 60$ years & $2.58(1.25$ to 4.64$)$ & 0.30 & 0.001 \\
\hline (ii) Highest educational attainment: more than secondary schooling & $2.30(1.05$ to 5.01$)$ & 0.40 & 0.037 \\
\hline (iii) Diabetes knowledge: less than $\leq 7$ & $2.29(1.25$ to 4.20$)$ & 0.31 & 0.007 \\
\hline (iv) Psychological status, depressed (PHQ-2: $\geq 2$ ) & $2.30(1.30$ to 4.06$)$ & 0.29 & 0.004 \\
\hline
\end{tabular}

CI denotes confidence interval.

Nagelkerke $R^{2}=0.166$.

Hosmer-Lemeshow goodness-of-fit for the model: Chi-square $=11.635, \mathrm{df}=7$, and $p=0.113$.

(i) education; (ii) country of birth; (iii) glucose control as measured by $\mathrm{HbA}_{1 \mathrm{c}}$; and (iv) depression. In relation to educational attainment, those with up to primary schooling were more likely to have low health literacy (AOR: 3.12, 95\% CI: 1.17 to 8.30 ); conversely, those with postsecondary school were less likely to have low health literacy (AOR: 0.35, 95\% CI: 0.16 to 0.74$)$. Table 3 also shows that those born overseas were over two times (AOR: 2.17, 95\% CI: 1.21 to 3.91) more likely to have low health literacy; similarly, those who were depressed were also over two times (AOR: 2.01, 95\% CI: 1.12 to 3.59) more likely to have low health literacy. Unexpectedly, those with good glucose control, as indicated by $\mathrm{HbA}_{1 \mathrm{c}}$ of up to $7 \%$, had low health literacy (AOR: 0.41, 95\% CI: 0.29 to 0.90$)$. These four variables explained approximately $19 \%$ of the variance (Nagelkerke $R^{2}=0.191$ ), and HosmerLemeshow goodness-of-fit statistics was not significant (Chisquare $=2.937, \mathrm{df}=7$, and $p=0.891$ ), indicating good model fit.

3.3. Predictors of Low Diabetes Self-Management. Forward stepwise logistic regression analysis was likewise used to determine predictors of DSM, using the median of up to 35 as the cut-off score. Four variables emerged as independent and significant predictors of low DSM: (i) younger age group $(\leq 60$ years); (ii) having postsecondary schooling; (iii) low diabetes management knowledge score $(\leq 7)$, and (iv) being depressed (PHQ-2: $\geq 2$ ). The magnitude of the adjusted odds ratios was similar for all four predictor variables, ranging from 2.30 to 2.58 , explaining approximately $17 \%$ of the variance (Nagelkerke $R^{2}=0.166$ ). The Hosmer-Lemeshow goodnessof-fit statistics was not significant (11.635, $\mathrm{df}=7, p=0.113$ ), indicating good model fit (Table 4).

\section{Discussion}

In the current study, those with only primary school education, migrants, and those who reported depressed mood were more likely to have low health literacy. The relationship between education and health literacy has previously been reported [44]; while this was not an unexpected finding it was encouraging to find that participants with secondary schooling and above reported adequate health literacy. Further analysis of those with primary school education revealed that they were also more likely to be older (79\%) and overseasborn $(70 \%)$, which has important implications for targeting this group considering the demographic profile of the current study setting and its being a major area for immigrant settlement in Australia [23]. This is particularly important given that migrants have a disproportionately high prevalence of diabetes [45] and face a number of barriers such as limited English language proficiency, access issues, cultural beliefs, and socioeconomic factors that could have direct and indirect effects on health literacy and DSM [5, 45-47]. Compared with Australian-born participants, migrants in this study had significantly lower confidence in their ability to manage their diabetes $(p=0.019)$. Culturally tailored resources and lifestyle interventions addressing these barriers including fostering problem-solving skills, cultivating motivation by setting appropriate goals, and consistent follow-up could be important tools to build confidence for self-managing diabetes in this population.

Depression has been found to affect diabetes control through both physiological pathways [48], effects of treatment [49], and/or increasing demand for psychological and behavioural tasks involved in DSM [50]. Our study confirmed 
the finding that depressed mood and anhedonia are associated with low self-efficacy in carrying out DSM. The PHQ2 is sensitive, quick, and easily administered in a busy clinic setting which could allow for referral for psychological support. Given the negative influence of depression on diabetes control through several mechanisms, an important recommendation from this study would be that clinicians consider screening all patients who attend diabetes clinics for depression using the PHQ-2.

An unexpected outcome of our study was that poorer glucose control, as demonstrated by high $\mathrm{HbA}_{1 \mathrm{c}}$, was correlated with higher health literacy. This may be explained by two factors: the Health Literacy Scale used in this study measured general health literacy rather than health literacy specific to diabetes and therefore may not be suitable for the sample in this study. For example, one of the questions in this tool "How often do you have someone help you read hospital materials?" was answered by a number of participants with "never, because there was never anybody there to help me, I had to read them by myself," which reflected lack of support rather than a high level of health literacy. Secondly, having high health literacy may not necessarily translate into self-management actions that could result in better biochemical diabetes control. This contention is supported by the findings in this study that those who were highly educated had high health literacy but reported low DSM however; those who had higher diabetes knowledge score had higher DSM. A study on Englishspeaking adults with type 2 diabetes likewise found that health literacy (measured using S-TOFHLA) was not associated with $\mathrm{HbA}_{1 \mathrm{c}}$ or with the presence of diabetes complications [18]. In contrast, Schillinger et al. [12] found an association between low health literacy, poor diabetes control, and retinopathy in an ethnically diverse population.

Older participants in this study practiced more DSM although they had lower health literacy, perhaps because of heightened awareness of mortality whereby health becomes a main concern. It could also be that older participants spent more time engaging in DSM tasks as they had less external competing priorities compared with younger and more educated participants who, presumably, had job demands and family concerns which took priority over DSM. This study found no significant correlation between health literacy and DSM; however, it was lack of knowledge about diabetes specifically that predicted lower DSM.

A number of variables measured in this study were selfassessed constructs that were useful in illuminating the perceptions of participants regarding their resources in effecting DSM. For example, despite more than half of the participants' rating their health as good (53\%) and reporting adequate self-management (54\%), objective measurements of BMI and $\mathrm{HbA}_{1 \mathrm{c}}$ showed that a high number $(61 \%$ and $81 \%$, resp.) of participants were obese and/or had poorly controlled diabetes. This discordance between what participants perceived as "good" DSM and clinical parameters of good control is also consistent with previous studies of people with diabetes and other chronic conditions. For example, in a sample of rural Taiwanese residents Huang et al. [51] found that those who had $\mathrm{HbA}_{1 \mathrm{c}} \geq 7 \%$, indicating a poor level of control, assessed their health as good [51]. Large population-based studies have also demonstrated a "disconnect" between perceived and actual health in approximately one out of five individuals, with younger age, ethnicity (non-Hispanic Blacks), and higher socioeconomic status predicting this disconnect [52]. This discrepancy between self-perceived health status and objective measures of diabetes control is likely to have clinical implications for DSM education as improving the convergence between perceived and actual health may help promote self-management and, ultimately, improve health outcomes. It is therefore important for health professionals to stress the importance of maintaining a healthy weight and achieving optimal $\mathrm{HbA}_{1 \mathrm{c}}$ in patient diabetes education programs.

This study has several limitations. The participants were sampled from a cohort that is already accessing the diabetes clinics of two major centres in the region. This may not be representative of the general population with type 2 diabetes. Secondly, the study was cross-sectional and, given the chronicity of diabetes, a longitudinal study may have been more useful in assessing the effect of the variables under examination in relation to self-management over a period of time. Thirdly, the use of Chew's brief measure of health literacy may not accurately have reflected the level of health literacy in our participants. The tool had ceiling effects which may not be due to high health literacy. Another limitation of the study was the lack of recent (within the last three months) $\mathrm{HbA}_{1 \mathrm{c}}$ level, as some of the $\mathrm{HbA}_{1 \mathrm{c}}$ results used in this study were taken within the last two years (2014-2016) and, therefore, may not have been contemporaneous with data collection. Finally, as with all studies that collect data using selfreport measures, social desirability bias may have impacted on these findings and, given the discrepancy between selfreported health and $\mathrm{HbA}_{1 \mathrm{c}}$, this seems possible. Notwithstanding these limitations, this study presented findings that refute the relationship between health literacy and DSM in a culturally diverse urban population.

\section{Conclusion}

Sociocultural research exploring the factors affecting DSM is important to determine areas that may be amenable to implementing cost-effective interventions. In culturally diverse populations with T2DM, while sociocultural factors are determinants of health literacy, this study has demonstrated that it was not health literacy per se but having knowledge specific to diabetes that was more important in predicting the practice of DSM behaviours. Addressing the discordance in perception of health and objective measures of diabetes control in DSM education may improve patient compliance and monitoring. Importantly, the finding that depression was a significant predictor of both low health literacy and low DSM underscores the need for clinicians to screen for depression to ensure that people with T2DM are provided with appropriate support which in turn may enable them to engage in self-managing their condition.

\section{Ethical Approval}

Ethics approval was obtained from the South Western Sydney Local Health District (HREC/14/LPOOL/133). 


\section{Competing Interests}

The authors declare that they have no competing interests.

\section{Acknowledgments}

This project received funding support from the NSW Health Nursing and Midwifery Office, Nursing and Midwifery Strategy Reserve Initiatives. The researchers would like to acknowledge the support of Mr. Yousef Barham, Manager, and Ms. Balwinder Sidhu, Director, Multicultural Health Service, Dr. Ivan Kuo, staff specialist endocrinology, Department Head of the Macarthur Diabetes Service, Ms. Elizabeth Fletcher, credentialed diabetes educator, and Ms. Beverley Willmann, dietitian, and the staff of Liverpool Diabetes Service.

\section{References}

[1] P. Z. Zimmet, D. J. Magliano, W. H. Herman, and J. E. Shaw, "Diabetes: a 21st century challenge," The Lancet Diabetes \& Endocrinology, vol. 2, no. 1, pp. 56-64, 2014.

[2] S. F. Farsani, M. P. Van Der Aa, M. M. J. Van Der Vorst, C. A. J. Knibbe, and A. De Boer, "Global trends in the incidence and prevalence of type 2 diabetes in children and adolescents: a systematic review and evaluation of methodological approaches," Diabetologia, vol. 56, no. 7, pp. 1471-1488, 2013.

[3] D. Dabelea, E. J. Mayer-Davis, S. Saydah et al., "Prevalence of type 1 and type 2 diabetes among children and adolescents from 2001 to 2009," Journal of the American Medical Association, vol. 311, no. 17, pp. 1778-1786, 2014.

[4] R. Oza-Frank, R. Stephenson, and K. M. Venkat Narayan, "Diabetes prevalence by length of residence among US immigrants," Journal of Immigrant and Minority Health, vol. 13, no. 1, pp. 1-8, 2011.

[5] D. Maneze, B. Everett, S. Kirby, M. DiGiacomo, P. M. Davidson, and Y. Salamonson, "I have only little English': language anxiety of Filipino migrants with chronic disease," Ethnicity and Health, 2016.

[6] S. Hinder and T. Greenhalgh, “This does my head in. Ethnographic study of self-management by people with diabetes," BMC Health Services Research, vol. 12, article 83, 2012.

[7] M. A. Powers, J. Bardsley, M. Cypress et al., "Diabetes selfmanagement education and support in type 2 Diabetes: a joint position statement of the American Diabetes Association, the American Association of Diabetes Educators, and the Academy of Nutrition and Dietetics," The Diabetes Educator, vol. 41, no. 4, pp. 417-430, 2015.

[8] UK Prospective Diabetes Study (UKPDS) Group, "Intensive blood-glucose control with sulphonylureas or insulin compared with conventional treatment and risk of complications in patients with type 2 diabetes (UKPDS 33)," The Lancet, vol. 352, no. 9131, pp. 837-853, 1998.

[9] M. Baxter, R. Hudson, J. Mahon et al., "Estimating the impact of better management of glycaemic control in adults with Type 1 and Type 2 diabetes on the number of clinical complications, and the associated financial benefit," Diabetic Medicine, 2016.

[10] N. C. Onwudiwe, C. D. Mullins, R. A. Winston et al., "Barriers to self-management of diabetes: a qualitative study among lowincome minority diabetics," Ethnicity and Disease, vol. 21, no. 1, pp. 27-32, 2011.
[11] A. J. Ahola and P.-H. Groop, "Barriers to self-management of diabetes," Diabetic Medicine, vol. 30, no. 4, pp. 413-420, 2013.

[12] D. Schillinger, K. Grumbach, J. Piette et al., "Association of health literacy with diabetes outcomes," Journal of the American Medical Association, vol. 288, no. 4, pp. 475-482, 2002.

[13] D. A. Kindig, A. M. Panzer, and L. Nielsen-Bohlman, Health Literacy: A Prescription to End Confusion, National Academies Press, Washington, DC, USA, 2004.

[14] I. Poureslami, L. Nimmon, I. Rootman, and M. J. Fitzgerald, "Health literacy and chronic disease management: drawing from expert knowledge to set an agenda," Health Promotion International, 2016.

[15] M. K. Paasche-Orlow and M. S. Wolf, "The causal pathways linking health literacy to health outcomes," American Journal of Health Behavior, vol. 31, no. 1, supplement, pp. s19-s26, 2007.

[16] A. F. Jerant, M. M. von Friederichs-Fitzwater, and M. Moore, "Patients' perceived barriers to active self-management of chronic conditions," Patient Education and Counseling, vol. 57, no. 3, pp. 300-307, 2005.

[17] D. Nutbeam, "The evolving concept of health literacy," Social Science \& Medicine, vol. 67, no. 12, pp. 2072-2078, 2008.

[18] N. S. Morris, C. D. MacLean, and B. Littenberg, "Literacy and health outcomes: a cross-sectional study in 1002 adults with diabetes," BMC Family Practice, vol. 7, no. 1, article 49, 2006.

[19] F. Al Sayah, S. R. Majumdar, B. Williams, S. Robertson, and J. A. Johnson, "Health literacy and health outcomes in diabetes: a systematic review," Journal of General Internal Medicine, vol. 28, no. 3, pp. 444-452, 2013.

[20] F. Rotella and E. Mannucci, "Diabetes mellitus as a risk factor for depression. A meta-analysis of longitudinal studies," Diabetes Research and Clinical Practice, vol. 99, no. 2, pp. 98-104, 2013.

[21] N. Schmitz, S. Deschênes, R. Burns, and K. J. Smith, "Depressive symptoms and glycated hemoglobin Alc: a reciprocal relationship in a prospective cohort study," Psychological Medicine, vol. 46, no. 5, pp. 945-955, 2016.

[22] J. Mc Sharry, F. L. Bishop, R. Moss-Morris, and T. Kendrick, "'The chicken and egg thing': cognitive representations and selfmanagement of multimorbidity in people with diabetes and depression," Psychology \& Health, vol. 28, no. 1, pp. 103-119, 2013.

[23] Australian Bureau of Statistics, Migration-Australia (3412.0) 2011-12 and 2012-13, ABS, Commonwealth of Australia, Canberra, Australia, 2013.

[24] Australian Bureau of Census, ABS Estimated Resident Population (ERP) ABS 3218.0 Regional Population Growth, Australia, 2012.

[25] Australian Bureau of Statistics, ABS Index of Relative SocioEconomic Disadvantage (SEIFA), ABS, 2006.

[26] Y. Salamonson, B. Everett, J. Koch, S. Andrew, and P. M. Davidson, "English-language acculturation predicts academic performance in nursing students who speak English as a second language," Research in Nursing \& Health, vol. 31, no. 1, pp. 86-94, 2008.

[27] K. Kroenke, R. L. Spitzer, and J. B. W. Williams, “The patient health questionnaire-2: validity of a two-item depression screener," Medical Care, vol. 41, no. 11, pp. 1284-1292, 2003.

[28] C. A. Eigenmann, T. Skinner, and R. Colagiuri, "Development and validation of a diabetes knowledge questionnaire," Practical Diabetes International, vol. 28, no. 4, pp. 166-170, 2011.

[29] K. Lorig, P. L. Ritter, F. J. Villa, and J. Armas, "Communitybased peer-led diabetes self-management: a randomized trial," The Diabetes Educator, vol. 35, no. 4, pp. 641-651, 2009. 
[30] A. Schmitt, A. Gahr, N. Hermanns, B. Kulzer, J. Huber, and T. Haak, "The Diabetes Self-Management Questionnaire (DSMQ): development and evaluation of an instrument to assess diabetes self-care activities associated with glycaemic control," Health and Quality of Life Outcomes, vol. 11, no. 1, article 138, 2013.

[31] L. D. Chew, K. A. Bradley, and E. J. Boyko, "Brief questions to identify patients with inadequate health literacy," Family Medicine, vol. 36, no. 8, pp. 588-594, 2004.

[32] T. Postmes, S. A. Haslam, and L. Jans, "A single-item measure of social identification: reliability, validity, and utility," British Journal of Social Psychology, vol. 52, no. 4, pp. 597-617, 2013.

[33] S. J. Dollinger and D. Malmquist, "Reliability and validity of single-item self-reports: with special relevance to college students' alcohol use, religiosity, study, and social life," The Journal of General Psychology, vol. 136, no. 3, pp. 231-242, 2009.

[34] J. M. Cortina, "What is coefficient alpha? An examination of theory and applications," Journal of Applied Psychology, vol. 78, no. 1, pp. 98-104, 1993.

[35] M. Tavakol and R. Dennick, "Making sense of Cronbach's alpha," International Journal of Medical Education, vol. 2, pp. 53$55,2011$.

[36] B. Löwe, K. Kroenke, and K. Gräfe, "Detecting and monitoring depression with a two-item questionnaire (PHQ-2)," Journal of Psychosomatic Research, vol. 58, no. 2, pp. 163-171, 2005.

[37] American Diabetes Association, "Standards of medical care in diabetes-2010," Diabetes Care, vol. 33, supplement 1, pp. S11S61, 2009.

[38] D. B. Sacks, D. E. Bruns, D. E. Goldstein, N. K. Maclaren, J. M. McDonald, and M. Parrott, "Guidelines and recommendations for laboratory analysis in the diagnosis and management of diabetyes mellitus," Clinical Chemistry, vol. 48, no. 3, pp. 436472, 2002.

[39] P. Peduzzi, J. Concato, E. Kemper, T. R. Holford, and A. R. Feinstem, "A simulation study of the number of events per variable in logistic regression analysis," Journal of Clinical Epidemiology, vol. 49, no. 12, pp. 1373-1379, 1996.

[40] IBM Corporation, IBM SPSS Statistics for Windows, Version 23.0, IBM Corporation, Armonk, NY, USA, 2013.

[41] D. P. Farrington and R. Loeber, "Some benefits of dichotomization in psychiatric and criminological research," Criminal Behaviour and Mental Health, vol. 10, no. 2, pp. 100-122, 2000.

[42] J. DeCoster, A.-M. R. Iselin, and M. Gallucci, "A conceptual and empirical examination of justifications for dichotomization," Psychological Methods, vol. 14, no. 4, pp. 349-366, 2009.

[43] J. W. Graham, "Missing data analysis: making it work in the real world," Annual Review of Psychology, vol. 60, pp. 549-576, 2009.

[44] I. van der Heide, J. Wang, M. Droomers, P. Spreeuwenberg, J. Rademakers, and E. Uiters, "The relationship between health, education, and health literacy: results from the dutch adult literacy and life skills survey," Journal of Health Communication, vol. 18, supplement 1, pp. 172-184, 2013.

[45] L. Montesi, M. T. Caletti, and G. Marchesini, "Diabetes in migrants and ethnic minorities in a changing world," World Journal of Diabetes, vol. 7, no. 3, p. 34, 2016.

[46] A. Nguyen and K. Edwards, "Barriers and facilitators of diabetes self-management: a qualitative study among vietnamese americans," Online Journal of Cultural Competence in Nursing and Healthcare, vol. 4, no. 2, pp. 5-16, 2014.
[47] J. Hu, K. Amirehsani, D. C. Wallace, and S. Letvak, "Perceptions of barriers in managing diabetes: perspectives of hispanic immigrant patients and family members," The Diabetes Educator, vol. 39, no. 4, pp. 494-503, 2013.

[48] P. J. Lustman, L. S. Griffith, R. E. Clouse et al., "Effects of nortriptyline on depression and glycemic control in diabetes: results of a double-blind, placebo-controlled trial," Psychosomatic Medicine, vol. 59, no. 3, pp. 241-250, 1997.

[49] R. R. Rubin, Y. Ma, D. G. Marrero et al., "Elevated depression symptoms, antidepressant medicine use, and risk of developing diabetes during the diabetes prevention program," Diabetes Care, vol. 31, no. 3, pp. 420-426, 2008.

[50] L. Fisher, J. T. Mullan, M. M. Skaff, R. E. Glasgow, P. Arean, and D. Hessler, "Predicting diabetes distress in patients with Type 2 diabetes: A Longitudinal Study," Diabetic Medicine, vol. 26, no. 6, pp. 622-627, 2009.

[51] T.-T. Huang, S.-E. Guo, C.-H. Chang et al., "Misperception among rural diabetic residents: A Cross-Sectional Descriptive Study," Journal of Advanced Nursing, vol. 69, no. 4, pp. 927-934, 2013.

[52] P. D. Loprinzi, "Factors influencing the disconnect between self-perceived health status and actual health profile: implications for improving self-awareness of health status," Preventive Medicine, vol. 73, pp. 37-39, 2015. 


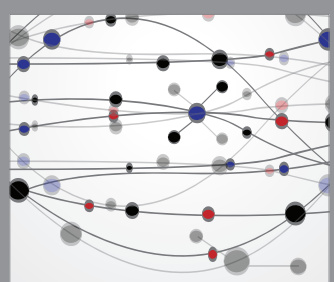

The Scientific World Journal
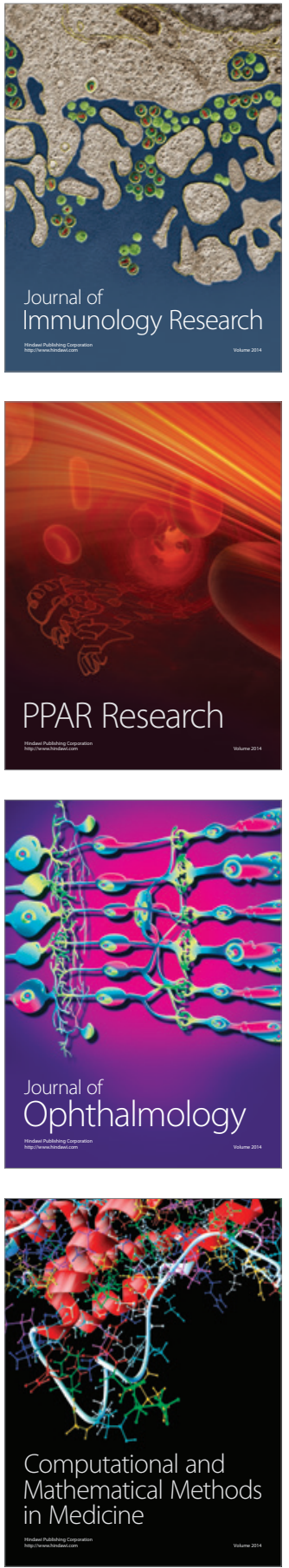

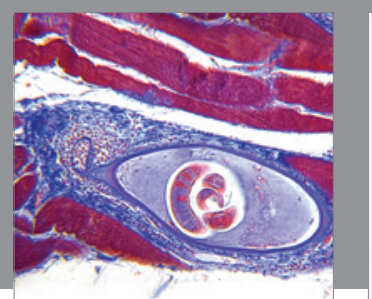

Gastroenterology Research and Practice

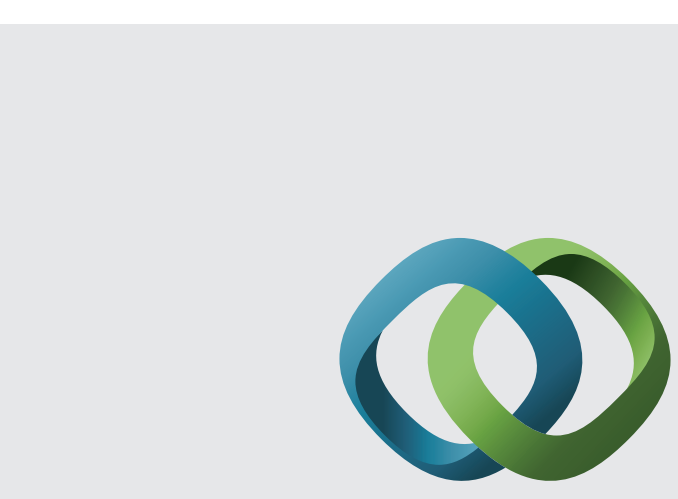

\section{Hindawi}

Submit your manuscripts at

http://www.hindawi.com
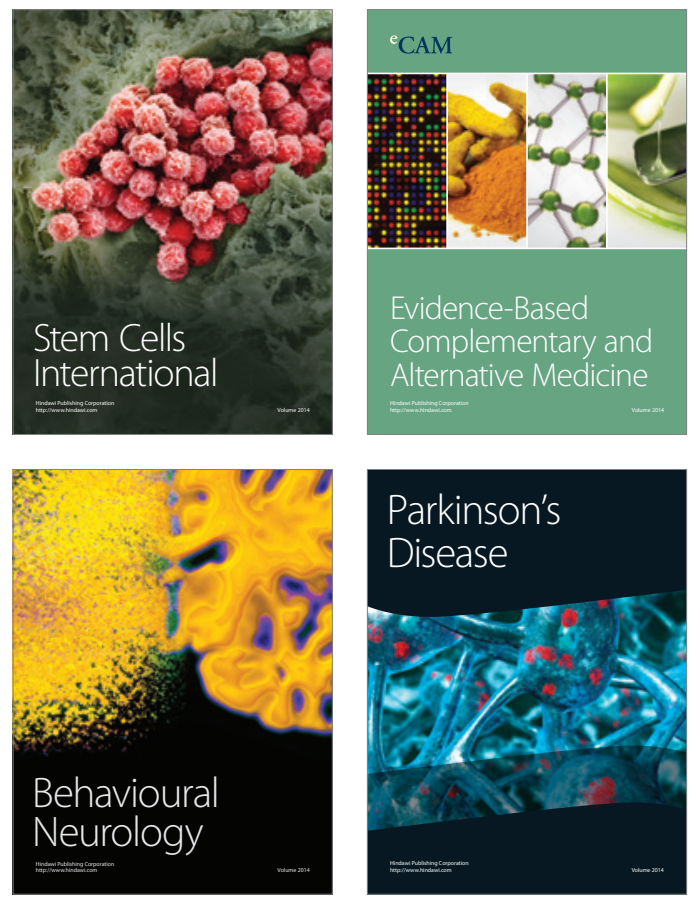
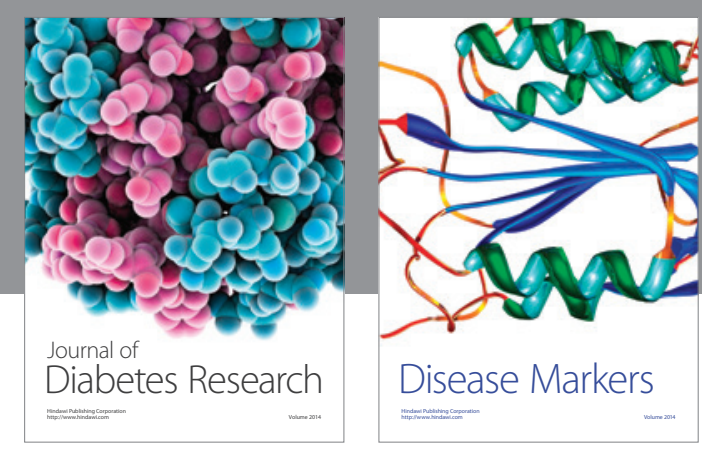

Disease Markers
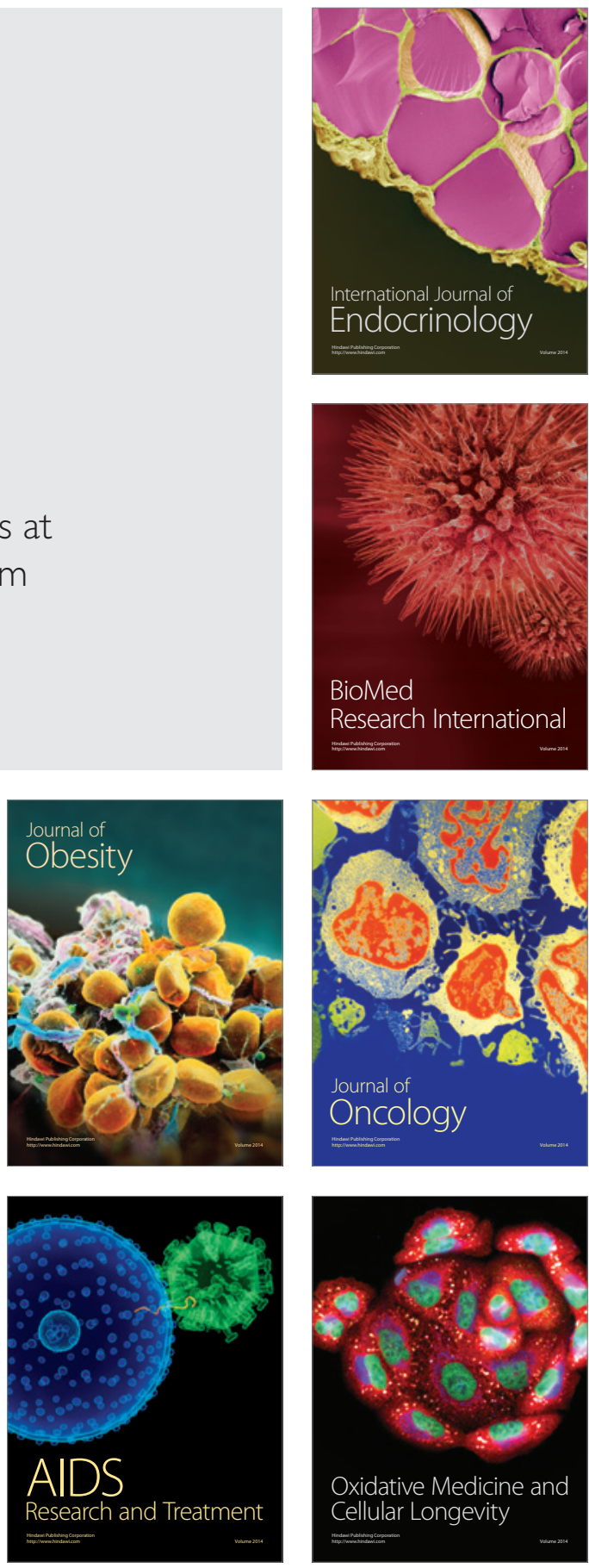